\title{
Socioeconomic Aspects of Spousal Concordance for Hypertension, Obesity, and Smoking in a Community of Rio de Janeiro, Brazil
}

\author{
Katia Vergetti Bloch, Carlos Henrique Klein, Nelson Albuquerque de Souza e Silva, \\ Armando da Rocha Nogueira, Lucia Helena Alvares Salis
}

Rio de Janeiro, RJ - Brazil

\begin{abstract}
Objective - The present study aims to evaluate the environmental role in the distribution of hypertension, obesity, and smoking and spousal concordance for the presencelabsence of these 3 cardiovascular risk factors.

Methods - A cross-sectional study was conducted in a community in Rio de Janeiro, Brazil. The households were randomly selected. Odds ratios were estimated to measure spousal concordance, across socioeconomic levels..
\end{abstract}

Results - Overall a significant aggregation of all 3 riskfactors was present. The crude odds ratiofor hypertension was $1.78(95 \% C I=1.02-3.08)$; for obesity, it was 1.80 (95\%CI=1.09-2.96); and for smoking, it was $3.40(95 \%$ $C I=2.07-5.61)$. The spousal concordance for hypertension decreased significantly $(p<0.001)$ from the lower to the higher educational level. In the case of obesity and smoking, the opposite was observed, although p-values for the linear trend were 0.10 and 0.08 , respectively.

Conclusion - In lower socioeconomic levels, couples are more concordant for hypertension and discordant for smoking. For hypertension and smoking, education seems to be a discriminant stronger than income, but for obesity the 2 socioeconomic indicators seem to represent different aspects of the environmental determinants of risk factor distribution.

Keywords: hypertension, obesity, smoking, risk factors, spouse concordance

Hospital Universitário Clementino Fraga Filho (UFRJ) and Escola Nacional de Saúde Pública. Fundação Oswaldo Cruz

Mailing address: Katia Vergetti Bloch - Rua Eurico Cruz, 47/501 - 22461-200 Rio de Janeiro, RJ - E-mail: kbloch@openlink.com.br
In 1998, cardiovascular diseases were responsible for $37.2 \%$ of adult deaths, excluding the ill-defined conditions in Brazil ${ }^{1}$. The social impact of this group of diseases is very high, with high economic costs, many premature deaths, and marked regional variations because of different levels of socioeconomic developmentin the country ${ }^{2,3}$.

Because many cardiovascular risk factors including hypertension, obesity, and smoking are determined by a combination of genetic, environmental (socioeconomic) or behavioural (life style) characteristics, or both, they are likely to cluster in families and in households ${ }^{4}$. Household aggregation is the occurrence of a disease or a characteristic more frequently among the residents sharing the same house than in the general population. Although in twin studies, the 3 components may operate together, spouse pairs studies allow the investigation of determinants of disease related to behavioral characteristics that can be modified, because the couples are likely to share the same lifestyle and socioeconomic environment, but not close genetic inheritance.

Intervention programs targeting the family are based on the belief that changes in lifestyle related to some risk factors (smoking, eating, exercise) might be more likely to occur if addressed to the whole household than if focused only on the individual. The aggregation of risk factors within a household is likely to be important for compliance to the intervention, but the socioeconomic environment shared by the residents is also.

A consensus exists that among blood relatives (genetic inheritance) hypertension and obesity aggregate, and studies also show that parents' smoking habits influence teenagers' smoking behavior ${ }^{5-10}$. The issue of aggregation of these risk factors in couples is controversial.

Whether aggregation of cardiovascular risk factors differs or not with socioeconomic status is a relevant question considering the presence of a strong socioeconomic gradient in morbidity and mortality from cardiovascular diseases in Western countries, including Brazil ${ }^{11-14}$. 
None of the reviewed studies has addressed the question of social class gradient concerning spouse-pair aggregation of cardiovascular risk factors.

To investigate the aggregation of the 3 risk factors, spouse concordance will be estimated to determine the state where husbands and wives share the same status in relation to the risk factors (presence or absence).

Based on data from a cross-sectional study originally designed to investigate the prevalence of hypertension ${ }^{15}$, this article attempts to answer 2 questions: 1) Are spousal pairs in this population concordant for hypertension, obesity, and smoking habits?; 2) If concordance does exist in couples, does it differ by socioeconomic level?

The use of 2 socioeconomic measures, one ecological (income) and the other individual (education), to evaluate patterns of concordance was also be addressed.

\section{Methods}

The study population comprises all adults (20 years or older) living in the Administrative Area of Ilha do Governador, Rio de Janeiro. The households were randomly selected by a 3 -stage cluster sampling after stratifying the area into 3 levels based on the average income of the census sectors ${ }^{16}$.

The eligible residents were interviewed in their houses with a standardized questionnaire to collect sociodemographic data and information on behavior. Blood pressure, weight, and height were measured by using standardized procedures, and the inter- and intraobserver variability were evaluated during the training period ${ }^{15,17,18}$. Blood pressure was measured twice with a modified mercury sphygmomanometer appropriate for research ${ }^{15}$. The second of the measurements (20-30 minutes after the first one) was used in the analysis.

The unavailable households or those in which eligible people were absent at the time of the visit were contacted at least 3 times. Simplified questionnaires were sent by post to the houses where refusals occurred and also to those where the residents could not be found by the interviewers.

The criterion used for the definition of hypertension was the one recommended by the World Health Organization ${ }^{19}$, which defines as hypertensive everyone with a systolic blood pressure of $160 \mathrm{mmHg}$ or more and/or a diastolic blood pressure of $95 \mathrm{mmHg}$ or more. Individuals who were receiving antihypertensive treatment were automatically considered hypertensives regardless of their blood pressure levels.

Body mass index (BMI), defined as weight (kg) divided by height squared $(\mathrm{m})$, was used to measure the degree of obesity. Obesity was defined as BMI of more then $27 \mathrm{~kg} / \mathrm{m}^{220-22}$.

Individuals who smoked cigarettes regularly or occasionally were classified as smokers. Ex-smokers and never smokers were considered nonsmokers.

The pairs were classified according to 2 types of socioeconomic measures. The first socioeconomic indicator used was income level, an ecological measure used to stratify the study population in the sampling process. So, couples were classified into 3 strata according to the average income of the census sectors of the households ${ }^{16}$. The low stratum is the one in which the mean income of the census sectors was less than 7 minimum wages payments per month (one minimum wage was approximately US\$77), the medium was-that with a mean income between 7 and 14 minimum wage payments, and the high was equivalent to a mean income above 15 minimum wage payments. The second criterion classified the subjects according to their education: Individuals: low - no formal education; Medium - less than eleven years of education; High - 12 or more years of education. Couples: Low - both partners with less than 11 years of education; Mixed - 1 spouse with low and the other with high education; High - both partners with 12 or more years of education.

The prevalence of the risk factors with a 95 percent confidence intervals (95\% CI) was calculated for the different socioeconomic levels and standardized by using the direct method ${ }^{23}$ with the total sample population considered standard. Differences between the socioeconomic levels were tested with chi-square tests for a linear trend ${ }^{24}$.

Concordance of dichotomous risk factors within spousal pairs was measured with the odds ratio $(\mathrm{OR})$, with their respective $95 \%$ confidence intervals ${ }^{25}$. OR greater than 1.0 indicates spousal concordance. Odds ratios were estimated by using logistic regression models, and the interaction of socioeconomic groups and the husband's risk factor was examined comparing the log likelihood under model (1) with that under model (2):

1) $\log$ (odds risk factor present in wife $)=\alpha+\beta E+\gamma_{1} v_{1}+$ $\gamma_{2} \mathrm{v}_{2}+\gamma_{3} \mathrm{v}_{3}$

2) $\log$ (odds risk factor present in wife) $=\alpha+\beta E+\gamma_{1} v_{1}$ $+\gamma_{2} \mathrm{v}_{2}+\gamma_{3} \mathrm{v}_{3+} \delta_{1} \mathrm{Ev} 1_{+} \delta_{2 \mathrm{Ev} 2}$

were, $a=$ intercept; $b=$ coefficient of risk factor in husband; $\mathrm{E}=0,1$ for absence/presence of the risk factor in husband; $\gamma_{1}$ and $\gamma_{2}=$ coefficients of dummy variables created for the socioeconomic groups 1 and $2 ; \mathrm{v}_{1}=$ dummy variable, created to represent the socioeconomic stratum $(1=$ medium stratum $0=$ others $) ; \mathrm{v}_{2}=$ dummy variable, created to represent the socioeconomic stratum $(1=$ high stratum $0=$ others $) ; \gamma_{3}=$ coefficient of the variable age; $\mathrm{v}_{3}=$ age $(1=$ less then 45 years; $2=45$ years or more); $\delta_{1 \text { and }} \delta_{2}=$ coefficients of interaction terms; Ev1 and Ev2 = terms that represent the interaction of social group and husbands' risk factor.

Scoring socioeconomic groups as 1, 2, 3 allowed testing for a linear trend in the odds ratios. The models were extended to adjust for age. Although the age was not the same for both members of the pair, it was very similar in practice (correlation, $r=0.89 \pm 0.02$ ), and it was possible to adjust for it because it was a shared covariate.

\section{Results}

From 674 households selected for the original study (prevalence of hypertension), 40 were unavailable (nobody answered after 3 visits) and 92 (13.7\%) refused to participate in the study. Among the 542 households participating in the 
study, 419 spouse pairs were identified, and in 370 (88.3\%) both members of the pair were interviewed and examined. Five pairs were excluded from the analysis because the wives were pregnant, leaving 365 couples.

The analysis of the available data for nonresponders showed that the proportion of married people is similar to that of the respondents.

The mean age of the population is 46.4 years, 48.7 for men and 44.7 for women. Table 1 presents the mean age for members of the couples according to the 2 socioeconomic indicators. Men and women in the lower income stratum are younger. When level of education is used, the situation is inverted. Men and women with low education are significantly older than those with high education. The individual classification of education shows that more women $(40.8 \%)$ than men $(33.4 \%)$ are in the lower level $(\mathrm{p}=0.04)$.

The age-adjusted prevalence of risk factors by sex and socioeconomic level are displayed in figure 1 . Women have higher prevalence rates of hypertension in lower socioeconomic levels than in higher for both socioeconomic indicators. Men are more obese and smoke less in higher income levels than in lower. The same trend was found for educational level, but statistical significance was borderline.

A spouse pair is concordant if both partners have the risk factor or if both do not have it, and, it will be discordant if one member has the risk factor and the other does not.

An overall association exists between husbands and wives for hypertension, obesity, and smoking habits (table 2 ). These associations produced similar results for the 2 socioeconomic indicators used. The adjustment for age tends to weaken associations, and for hypertension, a loss occurs in statistical significance.

Figure 2 displays the odds ratios and $95 \%$ CI for the 3 risk factors by socioeconomic level of the 2 indicators. Evidence of linear trends according to socioeconomic level is present only when the indicator is education. For hypertension, the magnitude of the association decreases from the lower to the higher socioeconomic level $(\mathrm{p}<0.001)$. On the other hand, some evidence exists that the concordance for obesity increases from the lower to the higher level al-

\begin{tabular}{|lccc|}
\hline \multicolumn{3}{|c|}{ Table I - Distribution and mean age of the pairs by socioeconomic } \\
level
\end{tabular}

though the test for a linear trend reaches a borderline statistical significance $(\mathrm{p}=0.08)$. The same pattern was found for smoking habits $(\mathrm{p}=0.10)$.

As previously noted, members of a couple can be concordant either because both have or because both do not have the risk factor. Table 3 presents the distribution of concordant pairs in relation to the 3 risk factors in each socioeconomic level. Concordant pairs are subdivided into concordant pairs for the presence of risk factors and concordant pairs for the absence of risk factors. The proportion of concordance in the absence of risk factors is given by the proportion of pairs where none of the partners has the risk factor in that strata, and the concordance in presence of risk factors is the proportion of pairs where both partners have it in that stratum. In relation to hypertension, the decrease in spousal concordance when we move from lower to higher levels of income or education was caused by the decrease in the proportion of concordant couples with the risk factor. But in the case of obesity, 2 different patterns are found depending on the socioeconomic index used. An increase occurs in the proportion of obese couples as income improves. On the other hand, an increase occurs in the proportion of pairs without obesity as educational level gets higher. Finally, it is observed that the proportion of nonsmoking pairs increases from lower to higher levels of income. When couples are stratified by educational level, more concordant pairs are present in the higher level than in the lower level.

\section{Discussion}

The present investigation showed an overall spousal pair concordance for hypertension, obesity, and smoking that is not homogeneous according to socioeconomic levels. These findings suggest that environmental factors, and not only genetic ones, may contribute to the determination of cardiovascular risk factors because concordance among couples may reflect similar life styles.

Although not a consensus in the literature, many studies have found similar results for blood pressure aggregation ${ }^{5,26-31}$. Spousal concordance for obesity and smoking have been investigated in fewer studies, and our results are in agreement with some $\mathrm{e}^{7,29-32}$ but not with others ${ }^{32-34}$. Many methodological differences among these studies as well as different cultural aspects of the populations studied may explain the controversial results.

Spousal concordance varied by socioeconomic level in different ways for the 3 risk factors, although statistically

\begin{tabular}{|lllll|}
\hline \multicolumn{4}{|c|}{$\begin{array}{c}\text { Table II - Crude (c) and age adjusted (a) overall odds ratios for } \\
\text { husband's and wife's hypertension, obesity, and smoking }\end{array}$} \\
\hline Risk Factor & OR (c) & $(95 \%$ CI $)$ & OR (a) & $(95 \%$ CI $)$ \\
\hline Hypertension & 1.78 & $(1.02-3.08)$ & 1.42 & $(0.83-2.43)$ \\
Obesity & 1.80 & $(1.09-2.96)$ & 1.72 & $(1.07-2.76)$ \\
Smoking & 3.40 & $(2.07-5.61)$ & 3.26 & $(2.03-5.24)$ \\
\hline
\end{tabular}




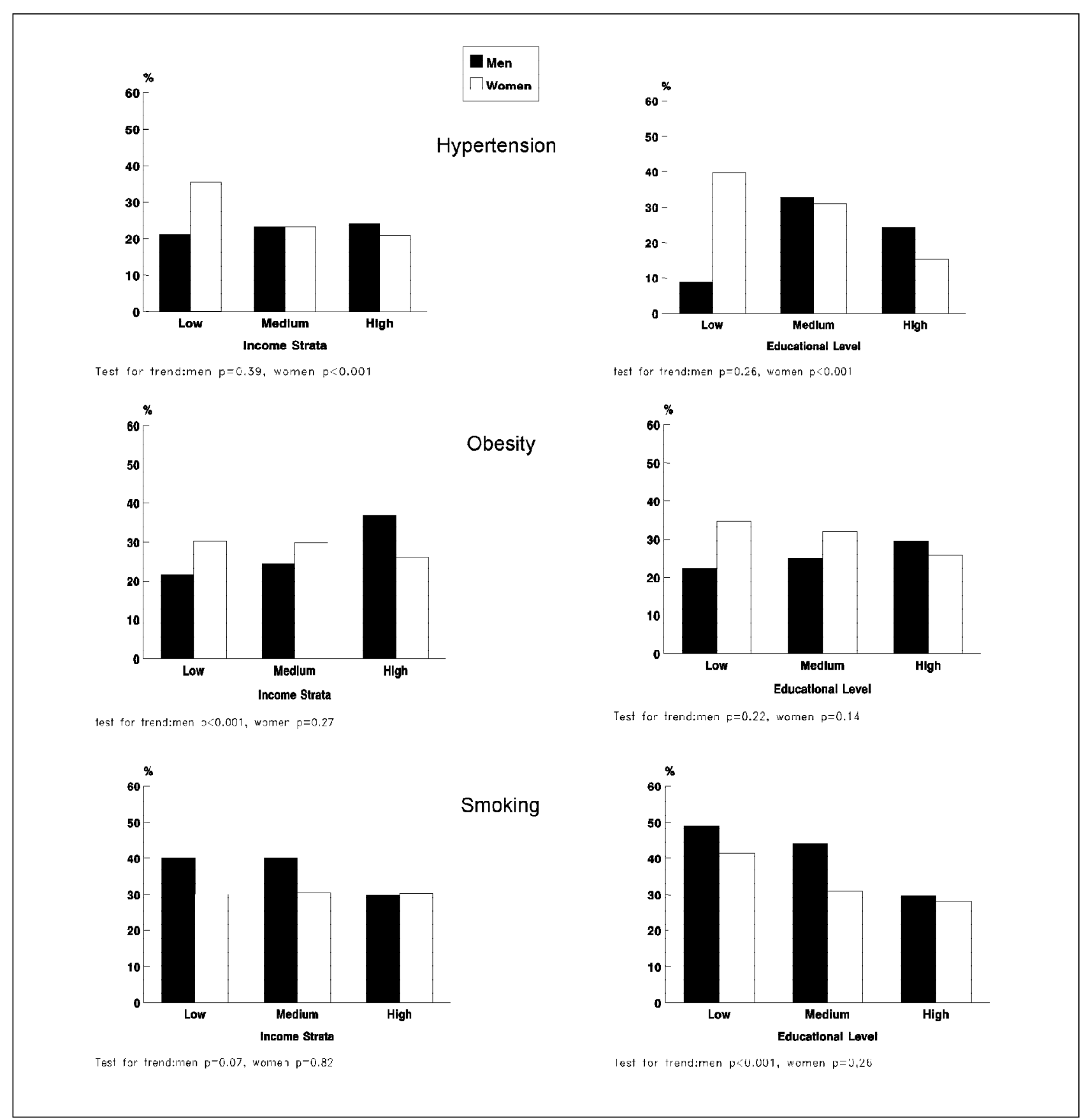

Fig. 1 - Age-adjusted prevalence rates of hypertension, obesity, and smoking by sex and socioeconomic level.

significant trends were found only when the socioeconomic category was defined based on educational level. It is important to note that none of the studies reviewed looked at the socioeconomic differences in spouse pair concordance for cardiovascular risk factors. The way concordant pairs are distributed among income and educational levels may contribute to the understanding of how environmental factors are operating in different groups.

The analysis of the concordance for hypertension by educational levels showed a statistically significant downward trend. The higher the level, the less concordant the spousal pairs. This pattern of aggregation may reflect a simi- lar individual behavior in partners with a lower educational background, and a poorer social environment may also influence it. Different mechanisms have been suggested to explain the negative association between hypertension and socioeconomic status, such as positive values and attitudes about health, access to a nutritious diet and health care, housing, and working conditions, and social support ${ }^{35-37}$.

Differently from hypertension, obesity had a significant upward trend in spousal concordance with the increase in the socioeconomic level. The proportion of obese pairs increases as the average income gets higher (table III). However, in the educational categories, a different pattern is observed and the 


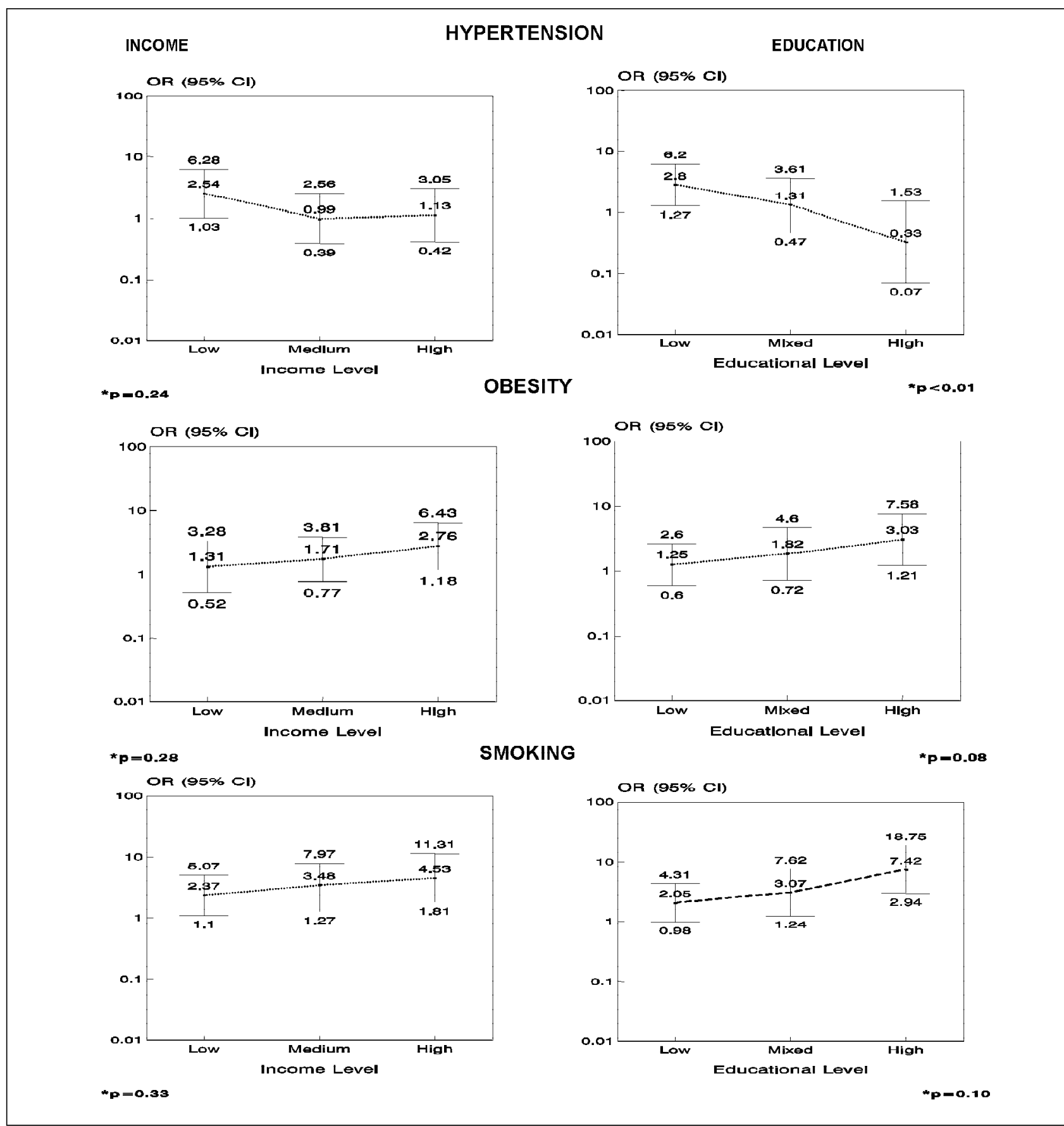

Fig. 2 - Age-adjusted odds ratios for spouse concordance of hypertension, obesity, and smoking by income and educational level. * p-value for linear trend.

increase in concordance is due to nonobese pairs. So, couples in the higher educational levels were similar because they were less obese. Here some evidence exists that the 2 socioeconomic indicators can represent different aspects of the environmental determinants of risk factor distribution. Although higher income may be directly associated with behavior patterns that induce obesity, higher educational levels may be associated with behavior patterns that reduce obesity among members of the same couple. In Hong Kong, it was shown that educational level is associated with a healthier diet and lower prevalence of overweight ${ }^{38}$.
Spouse similarity in dietary habits is probably present through all the socioeconomic levels. It was shown that in the lower strata, however, partners are likely to have different patterns of energy expenditures, with men having more physical activity related to work than women ${ }^{39}$. In the higher income strata, the imbalance between caloric intake and expenditure is probably similar for both sexes because men have more sedentary occupations. On the other hand, in the higher educational levels, men and women seem to have achieved a more balanced relationship between caloric intake and expenditure. 
Finally, the concordance in smoking habits increases from the lower to the higher levels because of an increase in the proportion of nonsmoking pairs (table III). In a study carried out in Finmark husbands and wives had similar smoking habits in $63.5 \%$ of all marriages ${ }^{40}$.

The overall odds ratios for spousal concordance of the 3 risk factors were reduced when adjusted for age but remained statistically significant, except for hypertension. Age-adjusted and crude estimates address different questions. The adjusted odds ratios avoid the occurrence of associations resulting from the confounding effect of age because some risk factors change with ageing and spouse pairs tend to be of similar in age. Differences in spousal concordance by socioeconomic levels could also occur due to a confounding effect of age because the age structures of the socioeconomic levels were different. Moreover, nonageadjusted odds ratios allow for a cumulative effect of the shared environment and are important in the identification of groups where spousal concordance is stronger and that could benefit from interventions focusing on couples rather than on individuals. For the purpose of identifying these groups, the confounding effect of age is not significant.

The concordance showed similar patterns of variations by the 2 socioeconomic indicators. In agreement with Abramson ${ }^{41}$, who found a good correlation among education and income indicators, these 2 socioeconomic variables were also highly associated in the present study $(\mathrm{p}<0.001)$. This fact can explain the reasonable consistence of the findings when both were used.

Education seems to be a stronger discriminant than income as we might expect because education is based on individual information and consequently tends to be more accurate than income, which is ecological information based on average values. Besides that, information on education is considered more reliable and more stable than information about income, and it can be measured independently for wives that are not in the work force ${ }^{42}$. In a Chinese population, education was the most important socioeconomic measure among the 4 indicators used (education, occupation, income, and marital status). People with a lower socioeconomic status had higher levels of cardiovascular risk factors in this study ${ }^{43}$. Also in Finland, Luoto et al ${ }^{44}$ found that education and occupation had a stronger association with a cardiovascular risk factor score in both men and women than income ${ }^{44,45}$.
The option to work with both indicators allowed the investigation of patterns of concordance that could be due to different mechanisms of determination. Studies have suggested that social and physical environments in poor areas of residence are associated with an increased risk of disease that depends on individual behavior ${ }^{13,36,42,46-48}$. On the other hand, the relation between education and disease has been associated with individual health practices. More educated people tend to make choices that are better for their health, independently from income.

In the present study, blood pressure levels used to classify individuals as hypertensives were higher than those used today. The WHO criteria $(160 / 95 \mathrm{mmHg}){ }^{19}$ was applied instead of the one proposed by the Joint National Committee on Prevention, Detection, Evaluation, and Treatment of High Blood Pressure (140/90) ${ }^{49}$.

If we use $140 / 90 \mathrm{mmHg}$ as the cutoff point for hypertension to look at the prevalences in the socioeconomic strata, we see that the trends do not change although the prevalence itself changes a lot. The pattern is the same for men and women and for both socioeconomic indicators, that is, prevalences are higher in the lower socioeconomic levels.

We also classified obesity by using as a cutoff point for BMI of $27 \mathrm{~kg} / \mathrm{m}^{2}$, instead of $30 \mathrm{~kg} / \mathrm{m}^{2}$, which is the WHO classification. We chose this cutoff point because many studies have shown that above this level an increase occurs in cardiovascular risk ${ }^{20-22}$. If we use the value $30 \mathrm{~kg} /$ $\mathrm{m}^{2}$, the trends change and the prevalences of obesity in the lower socioeconomic levels are higher than in the higher levels. Otherwise, if we use $25 \mathrm{~kg} / \mathrm{m}^{2}$ as the cutoff point for overweight, the trend pattern is quite similar to that seen before with $27 \mathrm{~kg} / \mathrm{m}^{2}$, that is, prevalences are higher in higher socioeconomic levels for both indicators, for men, but not for women.

The overall odds ratio for hypertension concordance decreases when we use the lower cutoff points (from 1.82 to 1.60 for the crude and from 1.42 to 1.13 for the adjusted). Nevertheless, the findings did not change. That is, adjustment for age caused a decrease in the odds ratio, which looses its statistical significance.

When we consider a higher cutoff point $\left(30 \mathrm{~kg} / \mathrm{m}^{2}\right)$ for obesity and when overweight as is investigated, the ORs continue to be statistically significant $\left(\mathrm{OR}_{\mathrm{c}}=1.77 ; \mathrm{OR}_{\mathrm{adj}}=\right.$

\begin{tabular}{|c|c|c|c|c|c|c|}
\hline & \multicolumn{2}{|c|}{ Hypertension } & \multicolumn{2}{|c|}{ Obesity } & \multicolumn{2}{|c|}{ Smoking } \\
\hline & Absence & Presence & Absence & Presence & Absence & Presence \\
\hline Income & $\%(\mathrm{n})$ & $\% \quad(\mathrm{n})$ & $\%(\mathrm{n})$ & $\%(\mathrm{n})$ & $\%(\mathrm{n})$ & $\%(\mathrm{n})$ \\
\hline Low & $58.5(72)$ & $11.4(14)$ & $54.5(67)$ & $8.1(10)$ & $42.3(52)$ & $19.5(24)$ \\
\hline Medium & $56.6(73)$ & $7.8(10)$ & $48.4(62)$ & 13.3 (17) & $49.6(64)$ & $16.3(21)$ \\
\hline High & $57.5(65)$ & $7.1(8)$ & $46.4(52)$ & 17 (19) & $56.6(64)$ & 16.8 (19) \\
\hline \multicolumn{7}{|c|}{ Education } \\
\hline Low & $53.6(82)$ & $14.4(22)$ & $45.1(69)$ & 12.4 (19) & $45.1(69)$ & $16.3(25)$ \\
\hline Mixed & $58(58)$ & $8(8)$ & 49.5 (49) & 14.1 (14) & $52(52)$ & $15(15)$ \\
\hline High & $62.5(70)$ & $1.8(2)$ & $56.8(63)$ & 11.7 (13) & $52.7(59)$ & 21.4 (24) \\
\hline
\end{tabular}


$1.92 ; \mathrm{OR}_{\mathrm{c}}=1.91 ; \mathrm{OR}_{\mathrm{adj}}=1.80$, respectively) confirming the concordance found with the $27 \mathrm{~kg} / \mathrm{m}^{2}$ cutoff point as a criterion for obesity.

The trends for the ORs across the socioeconomic levels are also similar for hypertension, obesity, and overweight when criteria are changed.

It is unlikely that the findings reported can be explained by selection bias. The information about the nonresponders obtained for the whole study population showed that, although they were more likely to be men, more educated and healthier (less medical services use), they were similar to the respondents concerning marital status, smoking habits, and hypertension diagnosis.

The knowledge of spousal concordance for cardiovascular risk factors and the understanding of its components may be helpful for planning studies to investigate different approaches for preventive interventions. Programs targeting individuals have been used and the effectiveness of such programs is variable ${ }^{50}$. The hypothesis that intervening in couples rather than in individuals may be more efficient has not yet been tested and the assessment of spouse concordance is a first step. In the British Family Heart Study ${ }^{51}$, men and women who benefit most from risk factor reductions have partners who also tend to benefit most, possibly through mutual reinforcement of lifestyle changes.

Hypertension is known to be an important cardiovascular risk factor and the estimated prevalence rates in Brazil vary from 10 to 40 percent $^{2}$. From a public health point of view, the spouse concordance for hypertension may be used as a strategy for case-finding because a man or a woman identified as hypertensive has almost a 2 times greater chance of having a partner that is also hypertensive than someone that is not. This aspect is particularly important for hypertension because it is usually symptomless until the late stages of the disease. The lower socioeconomic group (mainly the less educated) may be seen as a group more amenable to preventive strategies involving spouse pairs.

Preventive strategies towards obesity will require modification of cultural characteristics involving food preparation, eating, and physical activity that may not be easy to achieve. Perhaps couples are more likely to be motivated to change eating habits and to exercise than single individuals are.

Preventive strategies involving nonconcordant smoking couples and emphasizing the role of the nonsmoking partner may be important in programs to persuade people to stop smoking. Because discordant pairs are more likely to be in lower socioeconomic categories, actions targeting this group could be more effective.

Education seems to have a fundamental role in the determination of disease. Preventive strategies have to take into account the educational process as a whole, making it possible that all members of society have the same access to the information necessary to make healthy choices.

\section{Acknowledgements}

The authors thank Martin Bobak and Steve Pyke for their technical assistance and helpful suggestions. This research was supported by a grant from the Ministry of Health and from CNPq.

\section{Referências}

1. DATASUS. Ministério da Saúde. Web page www.datasus.gov.br. File accessed on October 10, 2001.

2. Lessa I, Mendonça GAS, Teixeira MTB. Doenças crônicas não-transmissíveis no Brasil: dos fatores de risco ao impacto social. Bol Oficina Sanit Panam 1996; 120: 389-412.

3. Laurenti R, Buchalla CM. Myths about cardiovascular diseases. Arq Bras Cardiol 2001; 76: 99-110.

4. Glueck CJ, Laskarzewski PM, Rao DC, Morrison JA. Familial aggregation of coronary risk factors. In: Connor WE, Bristow JD. Coronary Heart Disease. Philadelphia: J.B. Lippincott, 1985.

5. Hayes CG, Tyroler HA, Cassel J. Family aggregation of blood pressure in Evans County, Georgia. Arch Intern Med 1971; 128: 965-75.

6. Havlik RJ, Garrison RJ, Feinleib M, Kannel WB, Castelli WP, McNamara P. Blood pressure aggregation in families. Am J Epidemiol 1979; 110: 304-12.

7. Green G, Macintyre S, Patrick W, Ecob R. Like parent like child? Associations between drinking and smoking behaviour of parents and their children. $\mathrm{Br} \mathrm{J}$ Addict 1991; 86: 745-58.

8. Robinson WM, Borges-Osorio MR, Callegari-Jacques SM, et al. Genetic and nongenetic determinants of blood pressure in a Southern Brazilian sample. Genet Epidemiol 1991; 8: 55-67.

9. Sorensen TIA, Stunkard AJ. Does obesity run in families because of genes? An adoption study using silhouettes as a measure of obesity. Acta Psychiatr Scand 1993; 370(suppl.): 67-72.

10. Slyper A, Schectman G. Coronary artery disease risk factors from a genetic and developmental perspective. Arch Intern Med 1994; 154: 633-8.

11. Duncan BB, Schmidt MI, Achutti AC, Polanczyk CA, Benia LR, Maia AAG. Socioeconomic distribution of noncomunicable disease risk factors in urban Brazil: the case of Porto Alegre. Bulletin of PAHO 1993; 27: 337-49.
12. Piccini RX, Victora CG. Hipertensão arterial sistêmica em área urbana no sul do Brasil: prevalência e fatores de risco. Rev Saúde Pública 1994; 28: 261-7.

13. Marmot MG. Socioeconomic determinants of CHD mortality. Int J Epidemiol 1989; 14: S196-S202.

14. da Silva MA, Sousa AG, Schargodsky, H. Risk factors for acute myocardial infaction in Brazil. FRICAS Study. Arq Bras Cardiol 1998; 71: 667-75.

15. Klein CH, Souzae Silva NA, Nogueira AR, Bloch KV, Campos LHS. Hipertensão arterial na Ilha do Governador, Rio de Janeiro, Brasil. I. Metodologia. Cad Saúde Públ 1995; 11: 187-201.

16. Instituto Brasileiro de Geografia e Estatística (IBGE). Censo de 1980. Rio de Janeiro.

17. Habicht JP. Estandarización de métodos epidemiológicos quantitativos sobre el terreno. Bol Oficina Sanit Panam 1974; 76: 375-81.

18. Rose G. Standartization of observers in blood pressure measurement. Lancet 1965; 1: 673-4.

19. World Health Organization. Diet, nutrition and prevention of cardiovascular disease. Technical Report Series 7970. 1990.

20. Gray DS. Diagnosis and prevalence of obesity. Med Clin North Am 1989; 73: 1-14

21. Gus M, Moreira LB, Pimentel M, Gleisener ALM, Moraes RS, Fuchs FD. Associação entre diferentes indicadores de obesidade e pevalência de hipertensão arterial. Arq Bras Cardiol 1998; 70: 111-4.

22. National Institutes of Health Consensus Development Conference Statement Health Implications of Obesity. Ann Int Med 1985; 103(6 pt 2): 1073-7.

23. Khan HA, Sempes CT. Statistical Methods in Epidemioloy. Oxford: Oxford University Press, 1989.

24. Fleiss JL. Statistical Methods for Rates and Proportions. New York: John Wiley \& Sons, 1981. 
25. Kleinbaum DG. Logistic Regression. A Self-Learning Text. New York: Springer-Verlag, 1994.

26. Tseng W-P. Blood pressure and hypertension in an agricultural and a fishing population in Taiwan. Am J Epidemiol 1967; 86: 513-24.

27. Suarez L, Criqui M, Barret-Connor E. Spouse concordance for systolic and diastolic blood pressure. Am J Epidemiol 1983; 118: 345-51.

28. Patterson TL, Kaplan RM, Sallis JF, Nader PR. Aggregation of blood pressure in Anglo-American and Mexican-American families. Prev Med 1987; 16: 616-25.

29. Sackett DL, Anderson GD, Milner R, Feinleib M, Kannel W. Concordance for coronary risk factors among spouses. Circulation 1975; 52: 589-95.

30. Pérusse L, Leblanc C, Tremblay A, et al. Familial aggregation in physical fitness. coronary heart disease risk factors and pulmonary function measurements. Prev Med 1987; 16: 607-15.

31. Knuiman MW, Divitini ML, Welborn TA, Bartholomew HC. Familial correlations, cohabitation effects, and heritability for cardiovascular risk factors. Ann Epidemiol 1996; 6: 188-94.

32. Hunt SC, Hasstedt SJ, Kuida H, Barry MS, Hopkins PN, Williams RR. Genetic heritability and common environmental components of resting and stressed blood pressures, lipids, and body mass index in Utah pedigrees and twins. Am J Epidemiol 1989; 129: 625-38.

33. Rissanen A, Helliövara M, Aromaa A. Overweight and antropommetric changes in adulthood: a prospective study of 17.000 Finns. Int J Obes1988; 12: 391-401.

34. Kolonel L, Lee J. Husband-wife correspondence in smoking, drinking, and dietary habits. Am J Clin Nutr 1981; 34: 99-104.

35. Dressler WW, Santos JE, Viteri FE. Blood pressure, ethnicity, and psychosocial resources. Psychosom Med 1986; 48: 509-19.

36. Haan M, Kaplan G, Camacho T. Poverty and health: prospective evidence from the Alameda County Study. Am J Epidemiol 1987; 125: 989-98.

37. Kaplan BH, Salonen JT, Cohen RD, Brand RJ, Syme SL, Puska P. Social connections and mortality from all causes and from cardiovascular disease: prospective evidence from Eastern Finland. Am J Epidemiol 1988; 128: 370-80.

38. Woo J, Leung SS, Ho SC, Sham A, Lam TH, Janus ED. Influence of educational level and marital status on dietary intake, obesity and other cardiovascular risk factors in a Hong Kong Chinese population. Eur J Clin Nutr 1999; 53: 461-7.

39. Duncan BB, Schmidt MI, Polanczyk CA, Homrich CS, Rosa RS, Achutti AC Fatores de risco para doenças não-transmissíveis em área metropolitana na
Região Sul do Brasil: prevalência e simultaneidade. Rev Saúde Públ 1993; 27 : 143-8.

40. Brenn T. Adult family members and their resemblance of coronary heart disease risk factors: the Cardiovascular Disease Study in Finnmark. Eur J Epidemiol 1997; 13: 623-30.

41. Abramson JM, Gofin R, Habib J, Pridan H, Gofin J. Indicators of social class: a comparative appraisal of measures for use in epidemiological studies. Sci Med 1982; 16: 1739-46.

42. Liberator P, Link BG, Kelsey JL. The measurement of social class in epidemiology. Epidemiol Rev 1988; 10: 87-121.

43. Yu Z, Nissinen A, Vartiainen E, Song et al. Associations between socioeconomic status and cardiovascular risk factors in an urban population in China. Bull World Health Organ 2000;78: 1296-305.

44. Luoto R, Pekkanen J, Uutela A, et al. Cardiovascular risks and socioeconomic status: differences between men and women in Finland. J Epidemiol Community Health 1994; 48: 348-54.

45. Bosma H, Appels A, Sturmans F, Grabauskas V, Gostautas A. Educational level of spouses and risk of mortality: the WHO Kaunas-Rotterdam Intervention Study (KRIS). Int J Epidemiol 1995; 24: 119-26.

46. Feldman JJ, Makuc DM, Kleinman JC, Cornoni-Huntley J. National trends in educational differentials in mortality. Am J Epidemiol 1989; 129: 919-33.

47. Winkleby MA, Fortmann SP, Barrett DC. Social class disparities in risk factors for disease:eight-year prevalence patterns by level of education. Prev Med 1990; 19: 1-12.

48. Wing S, Barnett E, Casper M, Tyroler HA. Geographic and socioeconomic variation in the onset of decline of coronary heart disease mortality in white women. Am J Public Health 1992; 82: 204-09.

49. Joint National Committee on Prevention, Detection, Evaluation, and Treatment of High Blood Pressure. The sixth report of the Joint National Committee of Prevention, Detection, Evaluation, and Treatment of High Blood Pressure. Arch Intern Med. 1997; 157: 2413-46.

50. Bauer RL, Heller RF, Challah S. United Kingdom Heart Disease Prevention Project: 12-year follow-up of risk factors. Am J Epidemiol 1985; 121: 563-9.

51. Pyke SD, Wood DA, Kinmonth AL, Thompson SG. Change in coronary risk and coronary risk factor levels in couples following lifestyle intervention. The British Family Heart Study. Arch Fam Med 1997; 6: 354-60. 